\title{
Antithrombotic therapy for the secondary prevention of ischemic stroke related to atrial fibrillation: ESC/ACC/ACCP Guidelines
}

\author{
Mirza Dilic*, Alden Begic, Nirvana Sabanovic Bajramovic, Amina Bico, Osman Terzic \\ Clinical Center University of Sarajevo, Sarajevo, Bosnia and Herzegovina
}

\begin{abstract}
Aim of this article is to present the state-of-the-art antithrombotic therapy for the secondary prevention of cardioembolic stroke due to new ESC, ACC, and ACCP guidelines which are the key-opinion guidelines in atrial fibrillation (AF). The statements are based on CHADS2 scoring calculation as well as newly developed CHA2DS2-VASc.
\end{abstract}

Statements: for the patients (pts) with a history of ischemic stroke (IS) or TIA and AF, including paroxysmal AF, guidelines recommend oral anticoagulation (Grade IA), aspirin (Grade IB), or aspirin and clopidogrel (Grade IB). In pts with a history of IS or TIA and AF, guidelines suggest dabigatran $150 \mathrm{mg}$ bid over adjusted-dose VKA (INR 2.0-3.0) (Grade IIB). ESC guidelines recommend for pts with CHA2DS2VASc Score 2, VKA therapy (INR 2,0-3,0), or direct thrombin inhibitor dabigatran, or an oral direct $\mathrm{Xa}$ factor inhibitors - rivaroxaban or apixaban (Grade IA). In pts with a history of IS or TIA and AF, who are unsuitable for or choose not to take an oral anticoagulant, guidelines recommend dual therapy, aspirin and clopidogrel (Grade IB). Recommendation is that oral anticoagulation should generally be initiated within 1 to 2 weeks after stroke onset. Earlier anticoagulation can be considered for pts at low risk of bleeding complications i.e. patients with a small infarct burden and no evidence of hemorrhage on brain imaging. Delaying anticoagulation should be considered for pts at high risk of hemorrhagic complications - those with extensive infarct burden or evidence of significant hemorrhagic transformation on brain imaging. In patients with a history of noncardioembolic IS or TIA, guidelines recommend long-term treatment with aspirin
(75-100 mg once daily), clopidogrel (75 mg once daily), aspirin/extended-release dipyridamole $(25 \mathrm{mg} / 200 \mathrm{mg}$ bid), or cilostazol (100 mg bid) (Grade IA), VKA (Grade IB), the combination of clopidogrel plus aspirin (Grade IB), or triflusal (Grade IIB). Of the recommended antiplatelet regimens, guidelines suggest clopidogrel or aspirin/extended-release dipyridamole over aspirin (Grade IIB) or cilostazol (Grade IIC).

Conclusions: It is an ongoing trend that recommendations follow CHADS2 scoring calculation as well as CHA2DS2VASc Score. Standard of care is based on net clinical benefit i.e. balance between clinical/prevention benefit and safety profile. Still there is a question mark: what to suggest for patients who are unsuitable for oral anticoagulants, for reasons other than major bleeding risk.

KEYWORDS: antithrombotic secondary prevention, stroke, atrial fibrillation.

CITATION: Cardiol Croat. 2013;8(9):299.

Received: $27^{\text {th }}$ Jul 2013

*Address for correspondence: Klinički centar Univerziteta u Sarajevu, Bolnička 25, 71000 Sarajevo, Bosnia and Herzegovina..

Phone: + 38733297438

E-mail: mdilic@bih.net.ba

\section{Literature}

1. Camm AJ, Lip GY, De Caterina R, et al. 2012 focused update of the ESC Guidelines for the management of atrial fibrillation: an update of the 2010 ESC Guidelines for the management of atrial fibrillation—developed with the special contribution of the European Heart Rhythm Association. Europace. 2012;14(10):1385-413.

2. Anderson JL, Halperin JL, Alber NM, et al. Management of patients with atrial fibrillation (compilation of 2006 ACCF/AHA/ESC and 2011 ACCF/AHA/HRS recommendations): a report of the American College of Cardiology/American Heart Association Task Force on Practice Guidelines. J Am Coll Cardiol. 2013;61(18):1935-44.

3. Lip GY. Recommendations for thromboprophylaxis in the 2012 focused update of the ESC guidelines on AF: a commentary. J Thromb Haemost. $2013 ; 11(4): 615-26$. 